\title{
RDUS
}

Revue de DROIT

UNIVERSITÉ DE SHERBROOKE

Titre : $\quad$ DEUX THÈSES SUR LA DÉMOCRATIE ET LE CONSTITUTIONNALISME : LA SOUVERAINETÉ DU PEUPLE ET L'ENGAGEMENT PRÉALABLE

Auteur(s): $\quad$ LUC B. TREMBLAY

Revue : $\quad$ RDUS, 2011, volume 41, numéro 3

Pages: $\quad 583-605$

ISSN : $\quad 0317-9656$

Éditeur: $\quad$ Université de Sherbrooke. Faculté de droit.

URI : $\quad$ http://hdl.handle.net/11143/10312

DOI : $\quad$ https://doi.org/10.17118/11143/10312 
Page vide laissée intentionnellement. 


\title{
ARTICLE
}

\section{DEUX THĖSES SUR LA DÉMOCRATIE ET LE CONSTITUTIONNALISME : LA SOUVERAINETÉ DU PEUPLE ET L'ENGAGEMENT PRÉALABLE}

\author{
par Luc B. TREMBLAY*
}

Peut-on réconcilier la souveraineté du Peuple et le principe démocratique avec le constitutionnalisme, c'est-à-dire, une forme de "Rule of Men" avec une forme de "Rule of Law"? Si oui, comment les tribunaux canadiens s'y prennent-ils conceptuellement? Dans ce texte, l'auteur examine et critique deux théories influentes au Canada : la théorie de la "souveraineté du Peuple "et la théorie de l'" engagement préalable". Selon l'auteur, l'une des thèses avancées par la Cour suprême repose sur l'idée qu'une Constitution est conçue pour "habiliter" ou "constituer" la démocratie, et non pas pour la "limiter". Il s'ensuit que ce qui permet de réconcilier le constitutionnalisme avec la démocratie ne réside pas tant dans l'acte de volonté "originel "du Peuple souverain d'édicter une Constitution, mais dans le contenu des normes constitutionnelles reconnues par les tribunaux comme étant valides et dont l'objet et l'effet instituent un système de gouvernance démocratique. De plus, il s'ensuit qu'il appartient aux tribunaux de déterminer ultimement quelles normes constitutionnelles rendent la démocratie possible et légitime.

Can one reconcile the notion of sovereignty of a people and the principle of democracy with constitutionalism? In other words, is it possible to integrate a form of "Rule of Men" with a form of "Rule of Law"? If the answer is affirmative, how would Canadian courts conceptualize this integration? The writer undertakes a critical examination of two influential theories in Canada: the "sovereignty of the People " theory and the "precommitment "theory. In his opinion, one of the theses posited by the Supreme Court relies upon the notion that a Constitution is conceived in order to "inspire " or to "create " democracy, and not to "limit " it. Therefore, that which harmonizes constitutionalism with democracy does not reside in the original intent of a sovereign people to adopt a constitution, but in the actual content of the constitutional norms destined to institute a democratic system of governance, and which are recognized as valid by the courts. Moreover, it is for the courts to determine ultimately which constitutional norms render democracy both possible and legitimate.

* . Professeur titulaire à la Faculté de droit de l’Université de Montréal. 


\section{SOMMAIRE}

1. Première thèse : la souveraineté du Peuple............. 588

i) La thèse de la souveraineté du Peuple original ...... 588

ii) La souveraineté du Parlement............................. 590

iii) La démocratie dualiste ....................................591

iv) La thèse de la souveraineté du Peuple au Canada........................................................ 592

2. Deuxième thèse : la thèse de l'engagement

préalable .......................................................... 595

i) L'argument souverainiste................................ 595

ii) La Cour suprême et l'engagement préalable .......... 598

iii) L'engagement préalable, la démocratie et le 600

Conclusion 603 
La philosophie du droit pourrait probablement se résumer à un vaste débat sur l'opportunité et sur les moyens appropriés de limiter l'exercice arbitraire des pouvoirs des gouvernants. L'idée que les pouvoirs gouvernementaux puissent être limités par des normes supérieures remonte même à l'origine de la philosophie du droit, comme en témoigne la célèbre tragédie grecque Antigone de Sophocle ${ }^{\text {. }}$

À l'époque moderne, ce débat a été centré sur les pouvoirs absolus du souverain qui, par essence ou par définition, avait le pouvoir arbitraire de décréter n'importe quelle loi sur n'importe quel sujet ${ }^{2}$. La souveraineté a d'abord été conçue comme un moyen de protéger les droits et les libertés des personnes, notamment la sécurité des personnes. Jean Bodin et Thomas Hobbes, par exemple, que l'on peut considérer être les pères de la théorie de la souveraineté, pensaient que sans l'institution d'un souverain qui jouissait de pouvoirs absolus et illimités, la société était condamnée au chaos et à l'anarchie ${ }^{3}$. Or, dans le chaos et l'anarchie, aucune sécurité n'est possible, aucun droit et aucune liberté ne peuvent être protégés. Il fallait donc l'institution d'un ordre juridique unique sanctionné et maintenu par la force. En ce sens, le droit, tel que voulu par le souverain, devait avoir la primauté; d'où l'idée de "Rule of Law". Cependant, puisque la volonté du souverain constituait la source ultime du droit, il était au-dessus du droit. Son pouvoir demeurait donc arbitraire d'un point de vue juridique et, comme on sait, plusieurs souverains européens n'ont pas hésité à l'exercer arbitrairement aussi d'un point de vue politique ou moral. D'où la réflexion sur l'opportunité et sur les moyens de limiter le pouvoir absolu du souverain.

En occident, deux principales stratégies ont été élaborées pour contrecarrer l'arbitraire des gouvernants. La première,

\footnotetext{
1. Antigone aurait été rédigée par Sophocle vers 441 avant JC.

2. Voir, par exemple, Gérard MAIRET, Le principe de souveraineté, Histoires et fondements du pouvoir moderne, Paris, Éditions Gallimard, 1997. D’où la réflexion sur les moyens de limiter les pouvoirs absolus du souverain.

3. Jean Bodin, Six livres de la République, 1576; Thomas HoBBEs, Léviathan, 1659.
} 
procédant des travaux de Jean-Jacques Rousseau, a consisté à conférer la souveraineté au Peuple lui-même ${ }^{4}$. La thèse est simple: si les gouvernés deviennent les gouvernants, alors les gouvernants vont agir dans l'intérêt des gouvernés et les droits des individus seront protégés : "le souverain n'étant formé que des particuliers qui le composent n'a ni ne peut avoir d'intérêt contraire au leur; par conséquent la puissance souveraine n'a nul besoin de garant envers les sujets, parce qu'il est impossible que le corps veuille nuire à tous ses membres, et nous verrons ci-après qu'il ne peut nuire à aucun en particulier» 5. C'est la thèse de la souveraineté du Peuple et celle de la démocratie classique. Le Peuple, ou le Demos, est souverain; il a donc le droit de se gouverner lui-même ("self government"). Il peut faire n'importe quelle loi sur n'importe quel sujet, conformément à sa conception du bien commun. Puisque sa volonté constitue la source ultime du droit, elle est au-dessus des lois et ne peut être limitée par aucune norme juridique, ni même celles de la constitution ${ }^{6}$.

La deuxième stratégie, procédant des travaux de John Locke et Montesquieu, a consisté à limiter les pouvoirs du gouvernement, quel qu'il soit, par des normes "constitutionnelles»" C'est la thèse du constitutionnalisme ${ }^{8}$. Son objectif est de protéger les droits fondamentaux et les libertés individuelles de type "libéral" à l'encontre des actions gouvernementales arbitraires. Une constitution n'a donc pas uniquement pour objet de créer, de structurer et de réglementer les pouvoirs du gouvernement. Elle doit aussi les "limiter" afin de protéger les droits fondamentaux et les libertés individuelles. La séparation des pouvoirs, une Charte des droits et le fédéralisme, par exemple, sont autant de mécanismes utiles.

4. Jean-Jacques Rousseau, Du contrat Social, Paris, Éditions GarnierFlammarion, 1966.

5. Id., à la p. 54 .

6. Id., à la p. 53 et aux p. 63-64.

7. John Locke, Deux traités du gouvernement, Paris, Vrin, 1997; Montesguieu, De l'esprit des lois, Paris, Garnier, 1973.

8. Comme doctrine politique, le constitutionnalisme apparaît principalement avec le libéralisme politique. 
Deux thèses sur la démocratie
(2011) 41 R.D.U.S. $\quad$ et le constitutionnalisme :
la souveraineté du peuple et l'engagement préalable

Ces deux stratégies caractérisent les idéaux de toutes les démocraties constitutionnelles contemporaines. Le problème, cependant, est qu'elles sont apparemment incompatibles. L'idéal démocratique valorise la primauté de la volonté (du Peuple) sur le droit, c'est-à-dire, une forme de "Rule of Men" ; l'idéal constitutionnel valorise la primauté du droit sur la volonté (du Peuple), c'est-à-dire, une forme de "Rule of Law". On oppose souvent ces deux idéaux comme un conflit entre la légitimité et la légalité du pouvoir. Mais cette représentation n'est pas tout à fait exacte: la légitimité et la légalité pourraient incarner deux modes concurrents de légitimation du droit.

La question qui se pose est de savoir si ces deux idéaux, la souveraineté du Peuple et le constitutionnalisme, la "Rule of Men" et la "Rule of Law", peuvent être réconciliés. Dans l'affirmative, comment le droit le fait-il au Canada? Comment le droit canadien peut-il s'incliner devant la souveraineté illimitée du peuple tout en valorisant le gouvernement limité? Peut-il être à la fois fidèle à la démocratie et au constitutionnalisme si ces deux idéaux pointent dans des directions opposées?

La question n'est pas que théorique. À chaque fois que les juges doivent décider si une loi du Parlement respecte les droits fondamentaux enchâssés dans la Constitution, ils doivent réconcilier les deux idéaux. De plus, les élus qui créent le droit se demandent souvent s'il est juste qu'il y ait des limites constitutionnelles à leur pouvoir ou s'ils ont, en principe, le droit de tout faire ce qu'ils veulent dès lors qu'ils sont majoritaires?

Il existe plusieurs théories destinées à montrer que le droit peut être fidèle à la fois à la démocratie et au constitutionnalisme. Je m'arrêterai aux deux théories qui semblent les plus influentes au Canada. Je les nommerai respectivement la thèse de la " souveraineté du Peuple " et la thèse de "l'engagement préalable". Je ne discuterai pas de la théorie du dialogue institutionnel, telle 
que reconnue par la Cour suprême du Canada dans certaines affaires ${ }^{9}$.

\section{Première thèse : la souveraineté du Peuple}

La première thèse a longtemps constituée la théorie dominante. Elle puise ses racines chez John Locke ${ }^{10}$. Elle se retrouve dans les Federalist Papers ${ }^{11}$ et dans la jurisprudence de la Cour suprême des États-Unis ${ }^{12}$. Les constitutionnalistes français de la Révolution l'ont rapidement acceptée ${ }^{13}$. Je la nommerai : la thèse de la souveraineté du Peuple.

\section{i) La thèse de la souveraineté du Peuple original}

En vertu de cette thèse, il y a une distinction entre le Peuple et le gouvernement. Le Peuple est souverain : ses pouvoirs sont donc illimités. Mais si le Peuple est souverain et que ses pouvoirs sont illimités, alors il peut agir en "Constituant". Il peut donc décider unilatéralement d'édicter une Constitution dont l'objet est d'établir le genre et la forme que doit prendre le gouvernement dont le but est de promouvoir le bien du Peuple. De ce point de vue, le genre et la forme de gouvernement importent peu. Mais dès qu'ils sont exprimés dans un acte constitutionnel, ils font autorité et le gouvernement institué est légitime dès lors qu'il agit en conformité avec la Constitution. Puisque le Peuple est souverain, il n'aliène jamais sa souveraineté ultime. Ce qu'il a créé, il peut le défaire à son bon plaisir.

9. J'ai examiné les limites des théories du dialogue dans Luc B. TremBlay, "Legitimacy of Judicial Review: The Limits of Dialogue between Courts and Legislatures", (2005) 3 The International Journal of Constitutional Law (ICON), 617-48.

10. Voir Locke, préc., note 7.

11. The Federalist Papers, N.Y., The New American Library Toronto, 1961.

12. Marbury $v$. Madison, 5 U.S. (1 Cranch) 137 (1803).

13. Voir, par exemple, Emmanuel-Joseph SIEYES, "Qu'est-ce que le Tiers état? ", dans R. ZAPPERI, Ecrits politiques, Paris, éditions des archives contemporaines, 1994. 
Aux États-Unis, c'est probablement le sens le plus influent du fameux "We, the People ". La Constitution américaine exprime la volonté souveraine du Peuple, telle que l'indiquait la voix des députés élus à cette fin dans chaque État, de se doter d'un gouvernement d'un certain type ${ }^{14}$. Comme le juge en chef Marshall de la Cour suprême des États-Unis l'a clairement exprimé dans l'affaire Marbury $v$. Madison ${ }^{15}$ : "That the people have an original right to establish for their future government such principles as, in their opinion, shall most conduce to their own happiness is the basis on which the whole American fabric has been erected» 16 .

Le gouvernement n'est donc pas le Peuple. Il ne détient pas la souveraineté. Il est un "agent" du Peuple souverain. Il constitue un ensemble d'institutions à qui le Peuple a délégué certains pouvoirs spécifiques dans la Constitution. Ces institutions doivent exercer leurs pouvoirs à titre de fiduciaire. Comme on l'écrivait dans les Federalist Papers : "The federal and State governments are simply agents and trusties of the people with different powers designed for different purposes»17. Puisque le gouvernement n'est pas souverain, ses pouvoirs peuvent être limités par l'acte constitutionnel. Le juge en chef Marshall exprimait cette idée en ces termes: "This original and supreme will organizes the government, and assigns, to different departments, their respective powers. It may either stop here, or establish certain limits not to be transcended by those departments»18.

Cette thèse est puissante. Elle montre comment le droit peut être fidèle à la fois à la démocratie et au constitutionnalisme. À chaque fois que le droit est appliqué conformément à la Constitution, il se conforme à la volonté souveraine du Peuple. De même, à chaque fois qu'un gouvernement agit à l'intérieur des limites imposées par la Constitution, il se soumet à la volonté du

14. Voir les Federalist Papers, préc., note 11, \# 39,

15. Marbury, préc., note 12.

16. Id., p. 176.

17. Voir les Federalist Papers, préc., note 11, \# 46.

18. Marbury, préc., note 12, p. 176. 
Peuple souverain. Quand un juge invalide une loi incompatible avec la Constitution, il maintient donc la démocratie : ce ne sont pas les juges, mais le Peuple qui a le dernier mot. Hamilton soutenait que le contrôle judiciaire des lois ne signifiait pas que "the judicial is superior to the legislative power. It only supposes that the people is superior to both". 19

\section{ii) La souveraineté du Parlement}

On pourrait soulever plusieurs objections à l'encontre de la thèse de la souveraineté du Peuple ${ }^{20}$. Je m'en tiendrai à une seule, l'objection fondée sur le principe de la souveraineté du Parlement et la démocratie majoritaire.

En pratique, les Constitutions des démocraties constitutionnelles contemporaines créent au moins une institution gouvernementale "démocratique ", telle qu'une assemblée parlementaire. Ces institutions sont censées représenter le Peuple. Il s'ensuit que les lois qu'elles édictent ou les politiques qu'elles sanctionnent sont censées exprimer la volonté du Peuple tel qu'il existe aujourd'hui. Il semble donc trompeur de soutenir que toutes les institutions gouvernementales ne constituent que des "agents" du Peuple qui agissent en vertu de pouvoirs délégués. Il existe généralement au moins une institution qui peut prétendre exprimer la " voix du Peuple souverain ".

Le cas échéant, le principe démocratique devrait impliquer que les lois du Parlement édictées à la majorité par les représentants élus du Peuple ont prépondérance sur la Constitution qui, du reste, n'exprime plus que la volonté d'un Peuple passé. Présupposer que la Constitution puisse avoir prépondérance sur les lois du Parlement d'aujourd'hui semble incompatible avec l'idéal démocratique. Pour cette raison, la

19. Voir les Federalist Papers, préc., note 11, \# 78.

20. J'ai examiné quelques objections dans Luc B. Tremblay, "General Legitimacy of Judicial Review and the Fundamental Basis of Constitutional Law ", (2003) 23 Oxford Journal of Legal Studies 525-62. 
Deux thèses sur la démocratie
(2011) 41 R.D.U.S. et le constitutionnalisme :
la souveraineté du peuple et l'engagement préalable

théorie du Peuple souverain ne semble pas conforme à la démocratie.

En vertu de cette objection, le modèle constitutionnel Britannique associé à la doctrine de la souveraineté du Parlement semble supérieur. En vertu de cette doctrine, le pouvoir gouvernemental suprême réside dans le Parlement (ou dans toute autre institution représentative) qui détient le contrôle direct et exclusif sur les lois, les actions gouvernementales et les actes de l'administration. Juridiquement, les pouvoirs du Parlement sont illimités: aucune norme constitutionnelle ne peut limiter son autorité et les tribunaux sont tenus d'appliquer toutes ses lois. En outre, puisque la Chambre des communes est le centre du pouvoir au Parlement et que les élus doivent rendre des compte devant l'électorat, la souveraineté du Parlement semble non seulement conforme au principe démocratique, mais aussi semble favoriser la souveraineté du peuple. Il permet au Peuple d'aujourd'hui de gouverner selon sa volonté; il lui permet de s'autogouverner21.

\section{iii) La démocratie dualiste}

Plusieurs auteurs aux États-Unis ont tenté de repousser l'objection fondée sur la souveraineté du Parlement en raffinant la thèse de la souveraineté du Peuple. En particulier, on a insisté sur le caractère dualiste de la démocratie constitutionnelle ${ }^{22}$. On a soutenu que, dans les faits, un Peuple pouvait s'exprimer de deux manières : tantôt comme Constituant et tantôt comme électorat. Lorsqu'il agit comme Constituant, le Peuple est souverain : il peut faire ce qu'il veut, y compris limiter les pouvoirs

21. Le modèle de la souveraineté du Parlement a été cristalisé par Albert Venn Dicey vers la fin du 19e siècle dans un chapitre de son traité consacré à cette question. Voir Albert Venn DICEY, Introduction to the Study of the Law of the Constitution, 10 $10^{\text {th }}$ ed., London, Macmillan Education Ltd., 1959.

22. Voir par exemple, Bruce ACKERMAN, "The Storrs Lectures: Discovering the Constitution ", (1884) 93 Yale L.J. 1013, at 1046; Bruce ACKERMAN, We the People: Foundations, Cambridge, Harvard University Press, 1991. 
des assemblées législatives censées le représenter. La forme même par laquelle il s'exprime importe peu. Toutes les formes sont bonnes, y compris les formes extérieures à la Constitution. Par contre, lorsqu'il agit comme électorat, il doit respecter la Constitution et, le cas échéant, les limites constitutionnelles qui lui sont imposées.

L'idée de force est que les jugements du Peuple Constituant sont plus substantiels, plus riches et plus réfléchis que les jugements temporaires d'une majorité électorale. Diverses raisons peuvent expliquer cette assertion, mais l'existence d'un jugement de type "constitutionnel " est toujours une question de fait. Il s'ensuit, en cas de conflit, que la Constitution doit avoir prépondérance sur les lois du Parlement. Certes cette forme de constitutionnalisme peut-être "anti-majoritaire " lorsque la Constitution limite les pouvoirs d'une majorité électorale temporaire qui voudrait porter atteinte aux valeurs et jugements réfléchis du Peuple. Cependant, elle n'est pas anti-démocratique, puisque la Constitution exprime la volonté du Peuple souverain. Le constitutionnalisme serait anti-démocratique s'il niait la voix du Peuple Constituant.

En vertu de cette réplique, une démocratie dualiste permet au droit d'être fidèle à la souveraineté du peuple tout en imposant des limites constitutionnelles aux institutions gouvernementales.

\section{iv) La thèse de la souveraineté du Peuple au Canada}

La thèse de la souveraineté du Peuple a été introduite au Canada par la Cour suprême au milieu des années 198023. Les raisons étaient assez particulières. La Constitution canadienne a été édictée à Londres par le Parlement du Royaume Uni. Elle n'est pas le produit d'un acte unilatéral posé par le Peuple canadien agissant en "constituant". Pouvait-on, dès lors, réconcilier le

23. J'ai examiné cette thèse dans Luc B. TREMBLAY, "Marbury v. Madison and Canadian Constitutionalism : Rhetoric and Practice", (2004) 36 The George Washington International Law Review 515 et dans (2003) 37 Thémis 375. 
Deux thèses sur la démocratie
(2011) 41 R.D.U.S. et le constitutionnalisme :
la souveraineté du peuple et l'engagement préalable

constitutionnalisme canadien avec l'idéal démocratique et l'idée de souveraineté du Peuple?

La Cour suprême a répondu oui, en adaptant la thèse de la souveraineté du Peuple à la situation canadienne. Pour la Cour, la Constitution avait été voulue par les "représentants élus du Peuple canadien". Ce fait, selon elle, rendait le droit constitutionnel conforme au principe démocratique et lui conférait toute sa légitimité. Dans le Renvoi sur la Motor Vehicle Act ${ }^{24}$, par exemple, le juge Lamer, pour une majorité, énonçait ceci :

Il ne faut pas oublier que la décision historique d'enchâsser la Charte dans notre Constitution a été prise non pas par les tribunaux, mais par les représentants élus de la population (people) canadienne. Ce sont ces représentants qui ont étendu la portée des décisions constitutionnelles et confié aux tribunaux cette responsabilité à la fois nouvelle et lourde. On doit aborder les décisions en vertu de la Charte en se libérant de tout doute qui peut subsister quant à leur légitimité25.

Ce thème a été répété à maintes reprises depuis. Lorsque les juges invalident une loi pour des motifs constitutionnels, ils n'imposent pas des limites incompatibles avec la souveraineté du Peuple. Ils exécutent les décisions du Peuple souverain, tel qu'il les a exprimées par ses représentants élus. Certes, ces décisions limitent l'action gouvernementale des mêmes représentants élus, mais il y a une distinction entre les représentants élus qui agissent en tant que constituant et les représentants élus qui agissent en tant que gouvernement. Lorsqu'ils agissent comme constituant, ils représentent le Peuple souverain. Ils peuvent faire ce qu'ils veulent, y compris limiter les pouvoirs des gouvernements. Lorsqu'ils agissent en tant que gouvernement, ils doivent respecter les limites imposées par la Constitution.

24. Renvoi sur la Motor Vehicle Act, [1985] 2 R.C.S. 486.

25. Id., à la p. 497. 
Dans le Renvoi relatif à la sécession du Québec ${ }^{26}$, par exemple, la Cour suprême du Canada a confirmé que les institutions gouvernementales, incluant les parlements, étaient tenues par les limites imposées par la Constitution :

Le principe du constitutionnalisme exige que les actes de gouvernement soient conformes à la Constitution. Le principe de la primauté du droit exige que les actes de gouvernement soient conformes au droit, dont la Constitution. Notre Cour a souligné plusieurs fois que, dans une large mesure, l'adoption de la Charte avait fait passer le système canadien de gouvernement de la suprématie parlementaire à la suprématie constitutionnelle. La Constitution lie tous les gouvernements, tant fédéral que provinciaux, y compris l'exécutif ... Ils ne sauraient en transgresser les dispositions: en effet, leur seul droit à l'autorité qu'ils exercent réside dans les pouvoirs que leur confère la Constitution. Cette autorité ne peut avoir d'autre source ${ }^{27}$.

Les représentants élus pourraient donc agir de deux manières : tantôt comme Constituant et tantôt comme gouvernements. Cette thèse dualiste permettrait de réconcilier le principe démocratique et le constitutionnalisme.

Cette thèse soulève plusieurs difficultés. En plus de tous les problèmes que pose la thèse de la souveraineté du peuple, elle en pose un plus spécifique : le Peuple souverain peut-il s'exprimer en tant que Constituant en dehors de ses représentants élus. Après tout, selon la thèse de la souveraineté du Peuple, c'est le Peuple lui-même qui est souverain, et non pas les représentants élus qui agissent en tant que Constituant. Du point de vue de cette thèse, les représentants élus qui agissent en tant que Constituant ne constituent qu'une institution gouvernementale parmi d'autres, bien qu'elle puisse être formellement plus fondamentale. Leurs pouvoirs n'ont formellement d'autres

26. Renvoi relatif à la sécession du Québec, [1998] 2 R.C.S. 217.

27. Id., par. 72 . 
Deux thèses sur la démocratie
(2011) 41 R.D.U.S. $\quad$ et le constitutionnalisme :
la souveraineté du peuple et l'engagement préalable

sources que la Constitution elle-même. La question est donc de savoir s'il reste quelque chose de substantiel dans l'idée que le Peuple souverain est distinct de la Constitution qu'il créé et des institutions gouvernementales qu'il institue et le demeure? Restet-il quelque chose de substantiel dans l'idée que le Peuple n'aliène jamais sa souveraineté ultime? C'est ce dont traite la seconde thèse.

\section{Deuxième thèse : la thèse de l'engagement préalable}

La deuxième thèse censée montrer que le droit peut être fidèle à la fois à la démocratie et au constitutionnalisme est plus complexe. Elle fait appel au concept d'«engagement préalable» (" precommitment"). La thèse de "l'engagement préalable" a été introduite au Canada dans le Renvoi relatif à la Sécession du Québec $^{28}$ pour repousser un argument avancé en faveur du droit du Peuple québécois de faire sécession sans respecter le processus d'amendement constitutionnel prévu dans la Constitution canadienne. Puisque cet argument a été avancé par les souverainistes québécois, je le nommerai "l'argument souverainiste".

\section{i) L'argument souverainiste}

L'argument souverainiste procède directement de la thèse de la souveraineté du Peuple (il constitue une application des thèses avancées par John Locke). Il énonce à peu près ceci. Puisqu'un Peuple souverain ne peut jamais aliéner sa souveraineté, il a toujours le pouvoir d'agir en tant que Constituant. Par conséquent, un Peuple souverain peut, non seulement faire une Constitution, il peut aussi unilatéralement décider de la défaire ou, à tout le moins, de s'en défaire. Autrement, le Peuple ne serait pas souverain. Il s'ensuit que le principe démocratique qui a conféré une légitimité à la Constitution canadienne devrait conférer la même légitimité à la décision volontaire du Peuple de s'en retirer.

28.

Id. 
La manière même d'exprimer la volonté du Peuple d'écarter un ordre constitutionnel donné peut prendre diverses formes, selon les contextes. Elle peut prendre la forme d'une convention, d'une assemblée constituante, d'une acclamation, d'une ratification, d'une opinion publique dominante, d'un rassemblement physique dans les rues et sur les places, et ainsi de suite. Ce qui est requis, tout en étant suffisant, est l'expression d'une volonté populaire directe, décidée et discernable.

L'argument souverainiste avançait que le Peuple pouvait exprimer sa volonté par référendum. Si la majorité du Peuple exprimait par référendum sa volonté de se dégager de l'ordre constitutionnel canadien afin d'établir une autre forme de gouvernement conformément à sa conception du bien commun, l'expression de cette volonté devait faire autorité. On pouvait peut-être débattre de la majorité requise: quel pourcentage? $50 \%+1,60 \%$ ? Mais le principe était clair. Un Peuple souverain ne peut jamais être dissout : il demeure toujours souverain, tant qu'il a la volonté d'exister politiquement, même si ce n'est que de façon latente. Tant que l'ordre constitutionnel est opérationnel, le Peuple est comme un volcan endormi. Il peut donc se réveiller à tout moment, dès lors qu'il estime que les fins suprêmes pour lesquelles il avait adopté une Constitution ne se sont pas réalisées ou se sont vues détournées.

La théorie du peuple ou de la nation élaborée par Sieyes pendant la Révolution française demeure l'une des sources intellectuelles les plus claires sur cette question :

un gouvernement n'exerce un pouvoir réel qu'autant qu'il est constitutionnel; il n'est légal qu'autant qu'il est fidèle aux lois qui lui ont été imposées. La volonté nationale, au contraire, n'a besoin que de sa réalité pour être toujours légale, elle est l'origine de toute légalité. Non seulement la nation n'est pas soumise à une constitution, mais elle ne peut pas l'être, mais elle ne doit pas l'être, ce qui équivaut encore à dire qu'elle ne l'est pas. ... De quelque manière qu'une nation veuille, il suffit qu'elle veuille; toutes les 
(2011) 41 R.D.U.S. Deux thèses sur la démocratie

formes sont bonnes, et sa volonté est toujours la loi suprême ${ }^{29}$.

L'argument souverainiste se heurtait toutefois à un obstacle important. La Constitution canadienne prévoyait elle-même expressément une formule d'amendement constitutionnel (voir les articles 38 et suiv.). Le Peuple Constituant, par ses représentants élus, n'avait donc pas seulement établi une forme de gouvernement, il avait aussi créé une institution habilitée à modifier les normes constitutionnelles : soit, selon le cas, la règle de l'unanimité des provinces ou celle exigeant sept provinces représentant au moins 50\% de la population. Je dirai de cette formule d'amendement qu'elle établit une "institution constituante".

Cette institution constituante pouvait-elle faire obstacle à la volonté du Peuple souverain de s'autodéterminer? Est-elle un nouveau pouvoir constituant qui a remplacé l'ancien pouvoir détenu par le Peuple? En vertu de l'argument souverainiste, la réponse était non. L'institution constituante devait aussi être conçue comme un "agent" du Peuple souverain, au même titre que toutes les institutions gouvernementales: elle n'est pas le Peuple. Le Peuple souverain est au dessus, à côté et antérieur à la Constitution, et cela signifie qu'il est indépendant des institutions habilitées à modifier la Constitution. Le Peuple souverain n'a jamais été dissous comme Peuple : comment pourrait-on du reste le faire disparaître? Sa souveraineté est un fait politique. Le Peuple peut donc, par référendum ou autrement, exprimer sa volonté d'écarter la Constitution, y compris l'institution constituante qu'elle crée.

La Cour suprême du Canada a rejeté l'argument souverainiste :

L'argument selon lequel on peut légitimement contourner la Constitution en s'appuyant sur un vote majoritaire obtenu dans un référendum provincial est

29. E-J. SiEYES, préc., note 13, aux p. 158-170, en particulier, à la p. 162. 


\begin{abstract}
superficiellement convaincant, dans une large mesure parce qu'il paraît faire appel à certains des principes qui sous-tendent la légitimité de la Constitution elle-même, c'est-à-dire la démocratie et l'autonomie gouvernementale. En bref, on avance que, puisque la notion de souveraineté populaire sous-tend la légitimité de nos arrangements constitutionnels actuels, alors cette même souveraineté populaire qui a donné naissance à la Constitution actuelle devrait aussi permettre au "peuple", dans l'exercice de la souveraineté populaire, de faire sécession par un vote majoritaire seulement. Une analyse plus poussée révèle toutefois que cet argument est mal fondé parce qu'il méconnaît le sens de la souveraineté populaire et l'essence même d'une démocratie constitutionnelle ${ }^{30}$.
\end{abstract}

L'argument de la Cour réside dans la thèse de l'engagement préalable.

\title{
ii) La Cour suprême et l'engagement préalable
}

La Cour suprême du Canada a repoussé l'argument souverainiste pour le motif qu'il méconnaissait "le sens de la souveraineté populaire et l'essence même d'une démocratie constitutionnelle ". Cette méconnaissance est mise à jour par la thèse de l'engagement préalable. Selon la Cour, un Peuple souverain a le pouvoir de s'engager pour l'avenir à respecter les règles d'une Constitution qu'il a lui-même fait adopter en tant que Constituant, y compris les règles qui définissent "qui" désormais exercera le pouvoir Constituant :

Un gouvernement constitutionnel est nécessairement fondé sur l'idée que les représentants politiques du peuple d'une province ont la possibilité et le pouvoir de prendre, au nom de la province, l'engagement pour l'avenir de respecter les règles constitutionnelles qui sont adoptées ${ }^{31}$.

30. Renvoi relatif à la sécession du Québec, préc., note 26, par. 75 .

31. Id., par 76. 
Deux thèses sur la démocratie

et le constitutionnalisme :

la souveraineté du peuple et l'engagement préalable

Un Peuple peut donc déterminer pour l'avenir comment il procédera pour faire des lois ordinaires, c'est-à-dire, comment il s'exprimera en tant que législateur, de même que comment il procédera pour amender la Constitution, c'est-à-dire, comment il s'exprimera en tant que pouvoir constituant. Par conséquent, le Peuple peut déterminer pour l'avenir quelle est la majorité requise pour édicter des lois ordinaires et quelle est la majorité requise pour amender les lois constitutionnelles.

Puisqu'il s'engage à respecter ces règles de la Constitution qu'il adopte, le Peuple souverain devient donc, à toutes fins utiles, ce que la Constitution établie : sa volonté ne peut plus s'exprimer que par l'ensemble des institutions gouvernementales, législatives, judiciaires et constituantes prévues par la Constitution. En ce sens, un Peuple souverain aurait le pouvoir de se redéfinir luimême pour l'avenir. Selon la Cour, cela signifie que les règles constitutionnelles "lient" les représentants politiques du peuple, mais elles les lient,

non pas en ce qu'elles font échec à la volonté de la majorité dans une province, mais plutôt en ce qu'elles définissent la majorité qui doit être consultée afin de modifier l'équilibre fondamental en matière de partage du pouvoir politique (y compris les sphères d'autonomie garanties par le principe du fédéralisme), de droits de la personne et de droits des minorités dans notre société ${ }^{32}$.

La thèse de l'engagement préalable permet de repousser l'argument souverainiste. Dès qu'un Peuple manifeste sa volonté d'instituer un gouvernement et une institution constituante, le Peuple s'est redéfini par l'ensemble de ces institutions. Le Peuple souverain, par le biais des représentants élus, aurait donc, en quelque sorte, abdiqué son pouvoir constituant originel en faveur de l'institution constituante qu'il a lui-même créée par la voix de ses représentants élus. Un Peuple souverain qui a la volonté d'exister politiquement s'institutionnalise et, à toutes fins utiles,

32. Id. 
se dissout en tant que puissance politique non institutionnelle. Le volcan ne s'est donc pas endormi : il s'est éteint.

Par ailleurs, il est intéressant d'ajouter que la thèse de l'engagement préalable permet aussi de repousser la thèse de la souveraineté du Parlement. Le Parlement n'incarne pas plus la volonté du Peuple souverain que l'institution constituante : il n'est qu'une institution gouvernementale parmi les autres et ne fait qu'exprimer les vues temporaires de l'électorat conformément à la règle de la majorité. Le Peuple souverain, je le répète, s'exprime par l'ensemble des institutions établies par la Constitution. Les règles constitutionnelles sont ce que le Peuple est devenu.

\section{iii) L'engagement préalable, la démocratie et le constitutionnalisme}

La thèse de l'engagement préalable se heurte aussi à plusieurs objections. La principale, pour nos fins, est que cette thèse semble enfermer tout le champ du politique dans le cadre formel de la Constitution. Il est donc légitime de se demander si cette thèse permet de réconcilier le constitutionnalisme et la démocratie d'une manière satisfaisante?

Certes, la Constitution canadienne établit des institutions législatives démocratiques et pose des limites à l'étendue de leurs pouvoirs. Cependant, ce fait est contingent. La thèse de l'engagement préalable ne requiert aucunement la création d'institutions démocratiques et n'exige aucune limite particulière aux pouvoirs gouvernementaux. Un Peuple pourrait donc s'engager d'avance à respecter des règles constitutionnelles qui conféreraient tous les pouvoirs législatifs à un groupe de prêtres et tout le pouvoir constituant à un général d'armée. De plus, il pourrait rendre les dispositions constitutionnelles impossible à modifier, ni même par l'institution constituante. Dès lors, le droit ne serait compatible ni avec l'idéal démocratique ni avec l'idéal constitutionnel. La légalité formelle (" le légalisme ") l'emporterait clairement sur la légitimité démocratique et le principe du constitutionnalisme. 
La Cour suprême du Canada est bien consciente de cette difficulté. C'est probablement pourquoi elle a proposé deux portes de sortie. La première est la plus connue. Elle réside dans les principes constitutionnels non écrits. Dans le Renvoi sur la sécession du Québec ${ }^{33}$, la Cour a reconnu l'existence de principes constitutionnels non écrits, dont le principe démocratique et le principe constitutionnel ${ }^{34}$. Bien interprétés, ces principes non écrits peuvent justifier des obligations constitutionnelles non écrites dont l'objet est d'honorer la démocratie et le constitutionnalisme. Ainsi, même si le Peuple originel s'est dissout dans la structure institutionnelle de la Constitution qu'il a adopté, le droit peut valoriser la démocratie et le constitutionnalisme. Par exemple, dans le Renvoi, le principe démocratique lu conjointement avec le principe du fédéralisme justifiait la reconnaissance d'une obligation constitutionnelle pour toutes les parties concernées de négocier avec le Québec, dès lors que le Québec, en tant que partie de la fédération, manifestait démocratiquement sa volonté de modifier la Constitution ${ }^{35}$. Certes, cette obligation était soumise à certaines conditions, dont l'existence d'une volonté claire de faire sécession ${ }^{36}$. Cependant, même si le Peuple originel s'est engagé à respecter la Constitution et s'est dissout lors de l'adoption de la Constitution, les populations provinciales contemporaines ont le droit d'exprimer démocratiquement leur volonté de modifier la Constitution. Lorsque cette volonté est exprimée clairement, elle fait naître pour toutes les parties concernées une obligation de négocier les termes de l'amendement proposé, sans garantie de résultat. Voici comment la Cour suprême s'est exprimée sur cette question :

Bien entendu, ces règles constitutionnelles sont ellesmêmes susceptibles de modification, mais seulement par un processus de négociation qui permet d'assurer à toutes les parties le respect et la conciliation des droits garantis par la Constitution. De cette façon, il est possible d'allier

33. Renvoi relatif à la sécession du Québec, préc., note 26 .

34. Id., aux par. 49-54, 61-69, 70-78.

35. Id., aux par. 83-88.

36. Id., aux par. 86-88. 
notre foi dans la démocratie et notre foi dans le constitutionnalisme. La modification de la Constitution requiert souvent quelque forme de consensus important, précisément parce que la teneur des principes fondamentaux de la Constitution l'exige. L'exigence d'un vaste appui sous forme de "majorité élargie" pour introduire une modification constitutionnelle garantit que les intérêts des minorités seront pris en considération avant l'adoption de changements qui les affecteront ${ }^{37}$.

La seconde porte de sortie réside dans le concept d'" habilitation". La Cour admet que la thèse de l'engagement préalable semble privilégier le constitutionnalisme au détriment de l'idéal démocratique. Cependant, nous aurions tort de le penser. Selon la Cour, le constitutionnalisme ne limite pas la démocratie. $\mathrm{Au}$ contraire, il "facilite et, en fait, rend possible un système politique démocratique en instaurant un cadre ordonné dans lequel les gens peuvent prendre des décisions politiques " ${ }^{38}$. En ce sens, une Constitution fournit le cadre institutionnel sans lequel le Peuple ne pourrait s'exprimer efficacement ni en tant qu'électorat ni en tant que Constituant. L'expression de la volonté du Peuple requiert une structure juridique qui lui permet d'exprimer sa volonté de manière cohérente.

La Cour n'explique pas beaucoup ce qu'elle a en tête par cette thèse. Néanmoins, elle semble penser que la Constitution " habilite " le Peuple à agir démocratiquement, elle ne le limite pas. La Constitution canadienne établirait les règles de la démocratie canadienne, un peu comme les règles d'un jeu. On aurait tort de penser que les règles du jeu d'échec, par exemple, qui déterminent comment la tour, le fou, les pions ou les cavaliers doivent agir, " limitent " la liberté de la tour d'avancer en diagonale, celle du fou de se déplacer verticalement, celle des pions d'aller dans tous les sens ou des cavaliers de sauter cinq cases à la fois. Ces règles ne limitent pas la "souveraineté" de la tour, du fou ou des pions. Elles ne limitent pas le jeu d'échec : elles "sont " le jeu d'échec.

37. Id., aux par. 76-77.

38. Id., au par. 78 . 
Deux thèses sur la démocratie
(2011) 41 R.D.U.S. $\quad$ et le constitutionnalisme :
la souveraineté du peuple et l'engagement préalable

Elles le rendent possible. Elles ne font pas que le réglementer : les règles du jeu sont constitutives du jeu.

Il en est de même d'une Constitution : on aurait tort de penser, par exemple, que les règles constitutionnelles "limitent" la démocratie ou la souveraineté du Peuple. Au contraire, les règles constitutionnelles rendent possible la démocratie et permettent au Peuple d'exercer sa souveraineté. Nulle institution ne peut logiquement, naturellement ou a priori revendiquer le droit exclusif de parler au nom du Peuple souverain. En même temps, ce dernier ne peut manifester sa volonté et s'autogouverner que par l'intermédiaire d'institutions reconnues coordonnées les unes aux autres, telles les institutions gouvernementales et constituantes reconnues dans une constitution.

Il découle de cette thèse que le constitutionnalisme ne devrait plus être conçu comme un ensemble de normes fondamentales qui "limite" la souveraineté du Peuple. On ne devrait plus le concevoir comme un principe négatif qui aurait pour objet de "refouler le politique", comme le soutenait Carl Schmitt. ${ }^{39}$ Le constitutionnalisme devrait être conçu comme un principe positif, un principe créateur de politique qui rend la démocratie possible.

\section{Conclusion}

La question de savoir si la démocratie et le constitutionnalisme sont réconciliables est complexe. Je tends à penser que l'argument fondé sur l'idée "d'habilitation ", tel qu'avancé brièvement par la Cour suprême du Canada dans le Renvoi sur la sécession du Québec ${ }^{40}$, pourrait constituer l'une de ses contributions les plus fécondes sur cette question. Il me semble que ce qui rend le constitutionnalisme compatible avec la démocratie réside, non pas dans le fait que le Peuple souverain ait édicté un jour, unilatéralement, une Constitution donnée, comme

39. Carl Schmit, Théorie de la Constitution, Paris, PUF, 1993, p. 172.

40. Renvoi relatif à la sécession du Québec, préc., note 26. 
le soutient la thèse du Peuple souverain, mais dans le fait que les normes constitutionnelles reconnues comme valides par les tribunaux " habilitent " un système de gouvernance démocratique.

Il s'ensuit, dans mon esprit, que les normes d'une Constitution ne devraient pas être conçues comme des «actes de volonté", ni même d'une volonté populaire originelle, mais comme des normes "rationnellement justifiées" par un appel à une conception normative d'un système de gouvernement démocratique. En ce sens, une Constitution n'est pas juste et bonne parce que le Peuple, à un moment donné de son histoire l'a voulue, mais le peuple d'aujourd'hui devrait la vouloir parce qu'elle maintient un gouvernement démocratique légitime.

J'ajouterais toutefois qu'il appartient aux tribunaux, pour des raisons que je ne peux pas explorer ici, de déterminer en dernier ressort quelles normes constitutionnelles rendent la démocratie possible ${ }^{41}$. Il leur appartient donc d'être les gardiens, non seulement de la Constitution, mais aussi de la démocratie canadienne et d'ajuster, à cette fin, le droit constitutionnel. La portée de cette assertion peut être très large et difficile à démontrer. Mais j'estime que le Renvoi sur la sécession du Québec, dans lequel la Cour a reconnu un ensemble de principes constitutionnels non écrits, justifiant la création d'une obligation de négocier une demande de modifier la Constitution soutenue par une majorité, l'illustre parfaitement. En outre, on pourrait même concevoir que les tribunaux puissent contrôler le contenu des amendements constitutionnels proposés ou édictés par une institution constituante (imaginons un amendement constitutionnel qui aurait pour objet de mettre fin à la démocratie). De plus, il appartient aux tribunaux de déterminer ce que signifie un système de gouvernance démocratique. Comme on sait, le concept de démocratie est contesté : est-ce une affaire

41. J'ai exploré ce thème dans Luc B. TREMBLAY, "General Legitimacy of Judicial Review and the Fundamental Basis of Constitutional Law ", préc., note 20. Voir aussi Luc B. TREMBLAY, "Les principes constitutionnels non écrits ", (2012) 17 Review of Constitutional Studies / Revue d'études constitutionnelles 15-51. 

Deux thèses sur la démocratie
(2011) 41 R.D.U.S. $\quad \begin{gathered}\text { et le constitutionnalisme : } \\ \text { la souveraineté du peuple et l'engagement préalable }\end{gathered}$

d'unanimité, de majorité, de droits fondamentaux? Si oui, lesquels? Seuls les droits politiques ou des droits non politiques?

La Cour doit trancher ces questions et je crois que c'est ce qu'elle fait en pratique. Si la conception que la Cour se fait de la démocratie était bien fondée ou raisonnablement acceptable ou si les normes constitutionnelles qu'elle reconnaissait contribuaient à l'institution d'une gouvernance démocratique, alors le droit canadien réconcilierait la démocratie avec le constitutionnalisme. 
